\title{
Dr. Seltsam, oder wie ich lernte, die Drohne zu lieben
}

\author{
Ein Überblick aktueller Debatten zum Drohneneinsatz
}

\author{
Karsten Weber, Institut für Sozialforschung und Technikfolgenabschätzung, Ostbayerische Technische Hochschule Regensburg, Galgenbergstraße 24, \\ 93053 Regensburg (Karsten.Weber@oth-regensburg.de), (D) orcid.org/0000-0001-8875-2386 \\ Bernhard Rinke, Zentrum für Demokratie- und Friedensforschung an der Universität Osnabrück (brinke@uni-osnabrueck.de) \\ Christian Alwardt, Institut für Friedensforschung und Sicherheitspolitik an der Universität Hamburg (alwardt@ifsh.de)
}

Drohnen werden inzwischen in vielen und sehr unterschiedlichen Kontexten verwendet. Aus dem Blickwinkel der Technikfolgenabschätzung (TA) scheint es daher sinnvoll, den Umfang der momentanen und zukünftigen Nutzung von Drohnen und daraus resultierende Implikationen näher zu beleuchten und eine Bestandsaufnahme durchzuführen. Darüber hinaus sollen die voraussichtlichen Pfade der weiteren Technikentwicklung, relevante Akteure und deren Interessenslage sowie zukünftige Anwendungspotenziale und Einsatzfelder analysiert werden.

Dr. Strangelove, or how I learned to stop worrying and love the drone A review of current debates on drone applications

Drones are nowadays used in many and very different contexts. From the technology assessment perspective, it therefore seems reasonable to shed more light on the extent of the current and future use of drones and the resulting consequences. In addition, the expected paths of further technological development, relevant actors and their interests as well as potential future applications and fields of use should be analyzed.

Keywords: drones, civil use, military use, technology assessment

\section{Militärische und zivile Nutzung von Drohnen}

Die Entwicklung von Drohnen begann bereits während des Ersten Weltkriegs; mindestens seit den 1960er-Jahren sind sie fester Bestandteil militärischer Operationen und spielen seit Anfang der 2000er-Jahre eine immer größere Rolle (Rogers und Hill 2014). Die Verwendung des Drohnenbegriffes ist dabei nicht auf unbemannte Luftfahrzeuge beschränkt, für spezielle Einsätze am Boden (bspw. Kampfmittelbeseitigung) oder im bzw. unter

This is an article distributed under the terms of the Creative Commons Attribution License CCBY 4.0 (https://creativecommons.org/licenses/by/4.0/)

https://doi.org/10.14512/tatup.27.3.11
Wasser (z. B. Seeminenabwehr) kommen ebenfalls unbemannte Systeme zum Einsatz. Die Handlungsautonomie dieser in Aufbau und Zielsetzung teils sehr unterschiedlichen Systeme ist derzeit noch limitiert und ihre Steuerung befindet sich regelmäßig unter menschlicher Kontrolle. Angesichts der intensiven Forschungs- und Entwicklungsaktivitäten ist jedoch zu erwarten, dass der Grad an Autonomie militärischer Systeme, die mit Robotik- und KI-Funktionen ausgestattet sein werden, ansteigt und ihre militärische Nutzung deutlich zunehmen wird.

Einerseits räumen Schlüsselstaaten wie die USA, China und Russland autonomen militärischen (Waffen-)Systemen in ihren politisch-strategischen Überlegungen sowie Forschungs- und Entwicklungsanstrengungen eine zunehmend hohe Priorität ein. Andererseits wird vor den ,,potenziell katastrophalen Konsequenzen“ (UN-Abrüstungschefin Izumi Nakamitsu) zukünftiger autonomer Waffensysteme gewarnt. Menschenrechtsorganisationen fordern ein Verbot autonomer Waffensysteme, da sie im Widerspruch zum humanitären Völkerrecht stünden, sich mit ihrer Einführung die Gefahr von Rüstungswettläufen ergeben und die Wahrscheinlichkeit militärischer Auseinandersetzungen steigen werde.

Ethische, politische, juristische, soziale und technische Fragen, die sich in Bezug auf den Einsatz militärischer Drohnen ergeben (Boyle 2015), betreffen z. B. die Proliferation bzw. deren Vermeidung, die Dual-Use-Problematik, die Nutzung durch substaatliche Akteure, das Problem der Krisenstabilität oder die Diskussion darüber, ob Drohnen militärische Konflikte wahrscheinlicher werden lassen. Darüber hinaus sind in Hinblick auf den Einsatz militärischer Drohnen Überlegungen zu ihrer technologischen Verlässlichkeit, Beherrschbarkeit oder Nachhaltigkeit (z. B. bei „Wegwerf“-Drohnen) anzustellen.

Derzeit eingesetzte Drohnen sind in aller Regel ferngesteuert, die Autonomie beschränkt sich auf das selbständige Navigieren zu einem Zielort oder die Fluglagesteuerung. Zukünftigen Drohnen werden vielleicht nur noch spezifische Einsatzziele vorgegeben, die diese dann selbständig verfolgen. Letztlich steht zur Debatte, ob Menschen die Entscheidung des Einsatzes von Gewalt treffen (humans in the loop) oder diese Entschei- 
dung autonom vom Waffensystem getroffen wird (humans out of the loop). Vor- und Zwischenstufen sind denkbar (humans on the loop), so dass diese ebenfalls zu identifizieren sind, da sich daraus unterschiedliche ethische und rechtliche Implikationen ergeben (Sharkey 2010). Hierauf aufbauend ist zu diskutieren, ob eine Regulierung oder Rüstungskontrolle autonomer bewaffneter Drohnen notwendig ist und wie diese umzusetzen wäre (Dickow et al. 2015).

In der Landwirtschaft finden Drohnen vor allem Anwendung bei der Kartierung landwirtschaftlicher Flächen (Broschart

\section{Angesichts der Herausforderungen}

$$
\text { und Probleme ihres zukünftigen }
$$

\section{Einsatzes wäre zu wünschen,}

\section{dass eine umfangreiche öffentliche}

\section{Debatte bezüglich Drohnen beginnt.}

2016) und bei Datenerhebungen zum Bodenzustand sowie zum Zustand von Pflanzenbeständen (Primicerio et al. 2012); dazu kommt die Überwachung von Herden (Favier et al. 2013). TA-relevante Fragen betreffen etwa aktuelle und zu erwartende Anwendungsmöglichkeiten in konkreten landwirtschaftlichen Produktionsverfahren, Akteure und deren Interessenlage, den Umfang der derzeitigen und zukünftigen Nutzung von Drohnen in der Landwirtschaft, voraussichtliche Pfade der weiteren Technikentwicklung, zukünftige Anwendungsfelder, Analyse und Bewertung von Anwendungspotenzialen, Forschungsbedarfe im Hinblick auf Technikentwicklung, Nutzung und Auswirkungen. Dabei sollte in den Blick genommen werden, ob Drohnen kleinräumige oder industrielle Landwirtschaftsformen unterstützen und welche Folgen die vorgenannten Anwendungen auf den Agrarsektor zeitigen. Von herausragendem Interesse sind überdies Fragen des Umweltschutzes und der Nachhaltigkeit (Danilov et al. 2015).

Als Transportmittel kommen Drohnen insbesondere bei der Auslieferung kleinerer Lasten infrage (Wrycza et al. 2017; Nentwich 2018). Allerdings wirft diese Nutzungsform zahlreiche rechtliche Fragen auf: Da existierende Versicherungsformen für Drohnen wohl nicht genutzt werden können, steht insbesondere die Versicherungswirtschaft vor erheblichen Herausforderungen (Großmann 2017).

Schließlich werden in der Literatur weitere zivile Einsatzzwecke, Technikfolgen jenseits des militärischen Einsatzfeldes sowie die juristische Gestaltung ziviler Nutzungen diskutiert (ein Beispiel unter vielen ist Rao et al. 2016). So erlauben Drohnen günstige Luftbildaufnahmen hoher Qualität, die für polizeiliche Zwecke genutzt werden können, aber Datenschutzfragen aufwerfen; vergleichbare Fragen stellen sich u. a. im Im- mobilienmarkt, wenn Privatgrundstücke überflogen und fotografiert werden. Nicht zuletzt sind Drohnen ein beliebtes Spielzeug - hier sind Fragen der Luftsicherheit, der Privatsphäre oder auch des Missbrauchs von Bedeutung.

\section{Die Beiträge dieses TATuP-Themas}

In den Beiträgen des vorliegenden TATuP-Themas können selbstverständlich nicht alle angesprochenen Aspekte behandelt werden. Dessen unbenommen erlauben die hier versammelten Texte gleichwohl einen Einblick in einige relevante Anwendungsund Problemfelder.

Susanne Benöhr-Laqueur behandelt ein aktuell umstrittenes Thema, das insbesondere in Bayern hohe Wellen geschlagen hat. Mit dem neuen bayerischen Polizeiaufgabengesetz (PAG) werden die Befugnisse der bayerischen Polizei neu geregelt, u. a. in Bezug auf den Einsatz von Drohnen. Die Autorin macht deutlich, dass die unklaren Formulierungen im PAG zu weitreichenden Konsequenzen für die Bürgerinnen und Bürger führen können; dabei wird sichtbar, dass Technikfolgenabschätzung sehr oft durch eine Rechtsfolgenabschätzung ergänzt werden sollte.

Markus Christen, Michel Guillaume, Maximilian Jablonowski, Peter Lenhart und Kurt Moll legen dar, dass in internationalen Debatten kein Konsens über die rechtliche Definition des Begriffes „Drohne“ besteht und dass eine Definition über technische Eigenschaften ungeeignet ist. Für das europäische Luftfahrtrecht schlagen sie daher eine alternative Beschreibung vor. Weiterhin gehen die Autoren auf technische Aspekte ein, die es erschweren, Drohnen in das Luftraumsystem einzubinden sowie auf die daraus erwachsenden ethischen, rechtlichen und sozialen Herausforderungen.

Jens Crueger beschreibt Chancen der Drohnennutzung für die archäologische Forschung. In Kombination mit diversen Nutzlasten könnten sie u. a. wertvolle Beiträge zur Dokumentation und bei der Erkundung von schwierigem Gelände leisten. Außerdem besitzen Drohnen das Potenzial, die Archäologie in Richtung eines minimalinvasiven Ansatzes zu verändern. Der Autor verschweigt dabei nicht die möglicherweise negativen Gefahren des Drohneneinsatzes insbesondere im Hinblick auf die unsachgemäße Nutzung durch SondengängerInnen sowie RaubgräberInnen.

Jessica Heesen, Susanne Schuster und Clemens Arzt wiederum untersuchen ethische und rechtliche Aspekte der gezielten Abwehr von Drohnen durch die Polizei. Da Drohnen nicht-intendiert abstürzen oder bewusst zum Absturz gebracht werden können, stellen sie eine Gefährdung für Menschen und Güter dar; Maßnahmen zur Abwehr müssen einer Risikoabschätzung und Güterabwägung unterzogen werden, bspw. um Verhältnismäßigkeit zu wahren. Die Autorinnen und der Autor untersuchen das Spannungsfeld unterschiedlicher moralischer und rechtlicher Ansprüche.

Bernhard Koch und Bernhard Rinke fragen in ihrem Beitrag, ob der militärische Einsatz von Kampfdrohnen gerecht- 
fertigt werden kann. Im Vordergrund steht dabei die Spannung zwischen dem Schutz der jeweils eigenen und der gezielten Tötung der gegnerischen Soldatinnen und Soldaten. Die Autoren argumentieren, dass der Einsatz von bewaffneten Drohnen moralisch bedenklich sei, da der Schutz, der auf der einen Seite für die eigenen Soldatinnen und Soldaten durch den Einsatz dieser Technologie verbessert werden mag, auf der anderen Seite mit einer erhöhten Gefahr für Leib und Leben einhergeht.

Simon Schneider, Bodo Bookhagen und Paul Eschbach liefern einen Übersichtsartikel, der auf das Potenzial der Drohnennutzung in verschiedenen Forschungsfeldern eingeht. Die Autoren betonen dabei, dass die Drohnennutzung im Forschungskontext nicht in einem rechtlichen oder regulatorischen Vakuum stattfindet, sondern bspw. Luftraumbeschränkungen oder Genehmigungsgefahren ausgesetzt ist. Damit wird erneut verdeutlicht, was bereits in Susanne Benöhr-Laqueurs Beitrag sichtbar wurde: Technikfolgen können ohne Berücksichtigung des regulatorischen Rahmens nur unzureichend abgeschätzt werden.

\section{Fazit}

Vollautonome Fahrzeuge befördern zwar Insassen, aber werden nicht mehr von Menschen gesteuert - insofern teilen sie viele Charakteristika mit Drohnen. Solche Fahrzeuge und die mit ihnen potenziell einhergehenden Folgen für Individuen und $\mathrm{Ge}$ sellschaften werden in diesem TATuP-Thema intensiv diskutiert. Angesichts der ebenso weitreichenden Konsequenzen des Drohneneinsatzes und der damit einhergehenden Herausforderungen und Probleme wäre zu wünschen, dass eine ähnlich umfangreiche öffentliche Debatte auch bezüglich Drohnen beginnt. Ansonsten könnten wir uns irgendwann in einer Situation wiederfinden, die jener ähnelt, die Stanley Kubrick in seinem Film beschreibt, auf den der Titel dieses Einführungskapitels anspielt.

\section{Literatur}

Boyle, Michael (2015): The legal and ethical implications of drone warfare. In: The International Journal of Human Rights 19 (2), S. 105-126.

Broschart, Daniel (2016): Potenziale des Drohneneinsatzes bei der räumlichen Bestandsaufnahme. In: AGIT - Journal für Angewandte Geoinformatik 2, S. 518-527, http://gispoint.de/fileadmin/user_upload/paper_gis_open/ AGIT_2016/537622068.pdf, zuletzt geprüft am 28.10.2018.

Danilov, A.; Smirnov, U.; Pashkevich, M. (2015): The system of the ecological monitoring of environment which is based on the usage of UAV. In: Russian Journal of Ecology 46 (1), S. 14-19.

Dickow, Marcel; Hansel, Mischa; Mutschler, Max (2015): Präventive Rüstungskontrolle. Möglichkeiten und Grenzen mit Blick auf die Digitalisierung und Automatisierung des Krieges. In: Sicherheit und Frieden (S + F) 33 (2), S. 67-73.

Favier, Marc-Alexandre; Green, Richard; Linz, Andreas (2013): The potential for UAV technology to assist in sheep management in the Scottish highlands. In: Bornimer Agrartechnische Berichte 81, S. 209-222.

Großmann, Henning (2017): Rechtliche und versicherungsrechtliche Probleme beim Einsatz von Drohnen. In: Transportrecht 40 (3), 98-105.
Nentwich, Michael; Horváth, Delila (2018): The vision of delivery drones. In: TATUP - Zeitschrift für Technikfolgenabschätzung in Theorie und Praxis 27 (2), S. 46-52.

Primicerio, Jacopo et al. (2012): A flexible unmanned aerial vehicle for precision agriculture. In: Precision Agriculture 13 (4), S. 517-523.

Rao, Bharat; Gopi, Ashwin Goutham; Maione, Romana (2016): The societal impact of commercial drones. In: Technology in Society 45, 83-90.

Rogers, Ann; Hill, John (2014): Unmanned. Drone warfare and global security. London: Pluto Press.

Sharkey, Noel (2010): Saying „no! " to lethal autonomous targeting. In: Journal of Military Ethics 9 (4), S. 369-383.

Wrycza, Philipp; Rotgeri, Mathias; ten Hompel, Michael (2017): Spielzeitreduktion autonomer Drohnen für den Transport eiliger Güter durch den Einsatz automatisierter Lastaufnahmemittel im Kontext eines ganzheitlich automatisierten Gesamtsystems. In: Logistics Journal: Proceedings, Vol. 2017. DOI: 10.2195/lj_Proc_wrycza_de_201710_01.

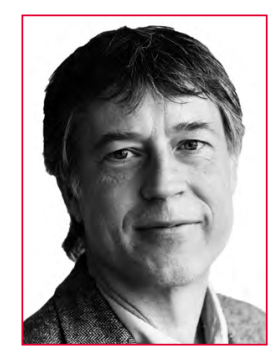

PROF.DR. PHIL. HABIL. KARSTEN WEBER ist Ko-Leiter des Instituts für Sozialforschung und Technikfolgenabschätzung (IST) der OTH Regensburg und einer der drei Direktoren des Regensburg Center of Health Sciences and Technology (RCHST) sowie Honorarprofessor für Kultur und Technik an der BTU Cottbus-Senftenberg. Er beschäftigt sich derzeit mit individuellen und gesellschaftlichen Auswirkungen von luk-Technologie sowie mit wertebasierter Gestaltung von Technik insbesondere im Gesundheitsbereich.

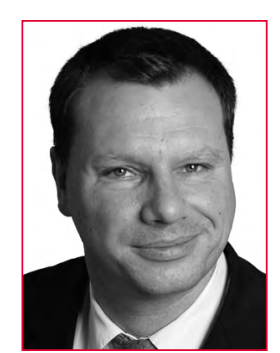

\section{DR. BERNHARD RINKE}

ist externer Projektmitarbeiter des Instituts für Theologie und Frieden in Hamburg, Mitglied des Zentrums für Demokratie- und Friedensforschung an der Universität Osnabrück sowie Lehrbeauftragter am dortigen Institut für Sozialwissenschaften. Aktuell beschäftigt er sich vor allem mit Fragen der ethisch legitimen Anwendung militärischer Gewalt. Unter dem Arbeitstitel „Ethische Fragestellungen im Kontext autonomer Waffensysteme" hat er gemeinsam mit Bernhard Koch vom Institut für Theologie und Frieden in Hamburg jüngst ein Gutachten für das Büro für Technikfolgen-Abschätzung beim Deutschen Bundestag verfasst.

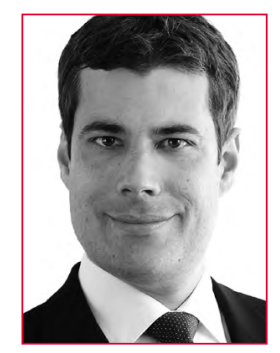

\section{DR. CHRISTIAN ALWARDT}

ist Diplom-Physiker und forscht am Institut für Friedensforschung und Sicherheitspolitik an der Universität Hamburg (IFSH) zu Fragen (inter-)nationaler Sicherheit und Stabilität. Ein Fokus liegt dabei auf der Technologiefolgenabschätzung und Rüstungskontrolle, mit besonderen Schwerpunkten auf unbemannten Systemen, der Automatisierung und den Implikationen einer zunehmenden Digitalisierung. 